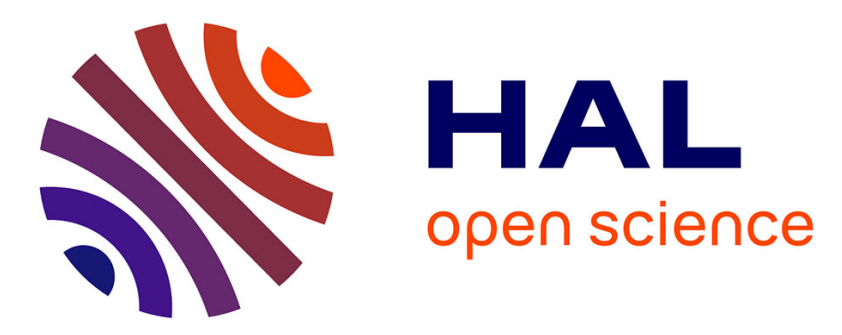

\title{
Small-Angle Neutron Scattering Reveals the Structural Details of Thermosensitive Polymer-Grafted Cellulose Nanocrystal Suspensions
}

Firas Azzam, Bruno Frka-Petesic, Enrico Semeraro, Fabrice Cousin, Bruno Jean

\section{To cite this version:}

Firas Azzam, Bruno Frka-Petesic, Enrico Semeraro, Fabrice Cousin, Bruno Jean. Small-Angle Neutron Scattering Reveals the Structural Details of Thermosensitive Polymer-Grafted Cellulose Nanocrystal Suspensions. Langmuir, 2020, 36 (29), pp.8511-8519. 10.1021/acs.langmuir.0c01103 . hal-02952428

\section{HAL Id: hal-02952428 \\ https://hal.science/hal-02952428}

Submitted on 13 Nov 2020

HAL is a multi-disciplinary open access archive for the deposit and dissemination of scientific research documents, whether they are published or not. The documents may come from teaching and research institutions in France or abroad, or from public or private research centers.
L'archive ouverte pluridisciplinaire HAL, est destinée au dépôt et à la diffusion de documents scientifiques de niveau recherche, publiés ou non, émanant des établissements d'enseignement et de recherche français ou étrangers, des laboratoires publics ou privés. 


\title{
Small Angle Neutron Scattering Reveals the Structural Details of Thermosensitive Polymer- grafted Cellulose Nanocrystals Suspensions
}

Firas Azzam, ${ }^{\dagger, 1}$ Bruno Frka-Petesic, ${ }^{\dagger, 2}$ Enrico F. Semeraro, ${ }^{\downarrow, 3}$ Fabrice Cousin, ${ }^{\S}$ and Bruno $\operatorname{Jean}^{\dagger} *$

${ }^{\dagger}$ Univ. Grenoble Alpes, Centre de Recherches sur les Macromolécules Végétales (CERMAV), F-38000 Grenoble, France

${ }^{\ddagger}$ Univ. Grenoble Alpes, CNRS, Grenoble INP (Institute of Engineering Univ. Grenoble Alpes), LRP, F-38000 Grenoble, France

${ }^{\S}$ Université Paris-Saclay, Laboratoire Léon Brillouin, Commissariat à l'Energie Atomique et aux Energies Alternatives (CEA) Saclay, F-91191 Gif-sur-Yvette, France

\begin{abstract}
Thanks to the use of small-angle neutron scattering (SANS), a detailed structural description of thermosensitive polymer-grafted cellulose nanocrystals (CNCs) was obtained and the behavior of aqueous suspensions of these derivatized biosourced particles upon temperature increase was revealed. Although literature data show that the surface grafting of thermosensitive polymers drastically enhance the colloidal properties of CNCs, direct space microscopic investigation techniques fail in providing sufficient structural information on these objects. In the case of CNCs decorated with temperature-sensitive polyetheramines
\end{abstract}


following a peptide coupling reaction, a qualitative and quantitative analysis of SANS spectra show that CNCs are homogeneously covered by a shell comprising polymer chains in a Gaussian conformation with a thickness equal to their radius of gyration in solution, thus revealing a mushroom regime. An increase of the temperature above the lower critical solution (LCST) of the polyetheramine results in the formation of finite size bundles whose aggregation number depends on the particle concentration and suspension temperature deviation from the LCST. SANS analysis further reveals local changes at the CNC surface corresponding to a release of water molecules and related denser polymer shell conformation. Noticeably, data show a full reversibility at all length-scales when a sample was cooled down below the LCST after being heated above it. Overall, results obtained by SANS allow an indepth structural investigation of derivatized CNCs that is of high interest for the design of functional materials comprising these biosourced colloids.

\section{Introduction}

Cellulose nanocrystals (CNCs) appear as unique rod-like biosourced colloids that are attracting growing attention from both the academic and industrial communities. The reasons for this interest are manyfold. From a material science point of view, CNCs display a range of highly valuable properties such as renewability, biodegradability, lightweight, nontoxicity and impressive mechanical properties with an axial elastic modulus estimated between 110 and $220 \mathrm{GPa}$ and further benefit from a recent intensification of their industrial-scale production. ${ }^{1-}$

${ }^{3}$ CNCs can also have variable dimensions that depend on their biological origin and to a lesser extent on the hydrolysis conditions, leading to a tunable aspect ratio, along with adjustable charge density and charge type, making them model colloids. ${ }^{4-5}$ From a colloidal science perspective, CNCs exhibit spectacular properties such as ability to be oriented in magnetic and electric fields, to stabilize emulsions and to self-organize into chiral nematic 
liquid crystals, leading to iridescence properties. ${ }^{6-13}$ All these characteristics make CNCs good candidates for a wide variety of applications, e.g. as reinforcing agents in nanocomposites or packaging, as rheology modifiers in cosmetics, paints or oil recovery, as well as additives in biomedical devices. ${ }^{14}$ In many cases, the implementation of additional CNC functionalities is required, such as stimuli-responsiveness or fulfilling compatibility conditions of CNCs with the solvent or matrix. To achieve this, a large range of surface functionalization strategies has been developed, mostly based on chemical modification or adsorption of molecular or macromolecular species. ${ }^{15-18}$ In this framework, we could show that the covalent grafting of thermosensitive polymer chains (Jeffamine ${ }^{\circledR}$ polyetheramine M2005, with a lower critical solution temperature, LCST, of about $16^{\circ} \mathrm{C}$ ) onto the surface of CNCs can be efficiently achieved using a peptide coupling strategy. Unlike their as-prepared counterpart, the resulting polymer-decorated CNCs exhibited a new range of properties: colloidal stability at high ionic strength in aqueous media, redispersion ability in organic solvents, amphiphilic character, thermoreversible and $\mathrm{pH}$-sensitive aggregation, and finally temperature-induced gelation. ${ }^{19-20}$ Importantly, this grafting maintained the self-organization properties into cholesteric phase and favored a smaller helical pitch. ${ }^{21}$

The characterization of the dimensions bare- and derivatized CNCs has become a major concern since the particle size and shape are key in a number of physical processes such as percolation or self-organization, directly impacting mechanical or optical properties. ${ }^{22}$ In the case of polymer-decorated CNCs, the object comprises a flexible polymer shell exhibiting a solvent-dependent conformation and surrounding a crystalline cellulosic core. The determination of the structural features, including dimensions and conformation, of this dual structure is a challenging task that requires specific tools. Indeed, conventional microscopic techniques such as transmission electron microscopy (TEM) or scanning electron microscopy (SEM) do not allow the visualization of the polymer corona in its hydrated state and can 
suffer from sample preparation artifacts. Even if cryo-TEM is able to reveal structural features of the objects in their suspended form, it is restricted to low-concentration samples and the lack of contrast prevents imaging the low-density polymer shell around the high-density cellulosic core. In contrast, scattering techniques such as small-angle neutron and X-Ray scattering techniques (SANS and SAXS) are well-suited to extract the detailed structural features of such hairy particles in the nano- to submicron length scale. SANS and SAXS also benefit from the possibility to probe samples in their original state in a very broad concentration range and under various experimental conditions. Moreover, these statistically relevant methods probe a large number of particles when compared to EM techniques. While SAXS and SANS have already been used to characterize the dimensions and the assembling or aggregation properties of as-prepared $\mathrm{CNCs},{ }^{23-37}$ they have been barely used to characterize coated or derivatized CNCs. ${ }^{38-40}$ In this work, we successfully used SANS to unravel the structural features of polyetheramine-grafted $\mathrm{CNCs}$, including the determination of the characteristic sizes as well as the grafted polymer shell conformation below and above its LCST. To this end, both a qualitative analysis based on the characteristic decays and quantitative fitting procedures based on models were used. The extent and reversibility of the temperature-induced aggregation were also investigated at multiple length scales.

\section{Experimental section}

Materials. Cotton linters were provided by Buckeye Cellulose Corporation and used as is. Jeffamine ${ }^{\circledR}$ polyetheramines M600, M2005 and M2070 were donated by Huntsman Corporation. These polymers are statistical amine-terminated copolymers of ethylene oxide (EO) and propylene oxide (PO). Table 1 summarizes the EO/PO ratio, molecular weight and LCST of the three polyetheramines used. Other chemicals were purchased from SigmaAldrich. Deionized water was used for all experiments. 


\section{Cellulose nanocrystals (CNCs)}

CNCs were obtained from the sulfuric acid hydrolysis of cotton linters with small modifications to the method described by Revol et al. ${ }^{41}$ Briefly, the cellulose was treated with 65 wt. \% sulfuric acid for 30 minutes at $63{ }^{\circ} \mathrm{C}$. The suspension was washed by centrifugation, dialyzed against distilled water, and sonicated for 4 min with a Branson model 450 sonifier at $30 \%$ intensity. After these treatments, the suspensions was filtered through $5 \square \mathrm{m}$ and then $1 \square \mathrm{m}$ cellulose nitrate membranes (Sartorius) and the resulting $2.3 \mathrm{wt} \%$ suspension was stored at $4{ }^{\circ} \mathrm{C}$. To avoid any bacterial contamination, some chloroform was added to the suspension. Conductometric titration and elemental analysis gave a sulfur content value equal $0.7 \%$. Combining these values with the size of the particles allowed us to estimate the average surface charge density as $0.48 \mathrm{e}^{-} / \mathrm{nm}^{2}$. A zeta potential of $-46 \square 5 \mathrm{mV}$ was determined with a NanoZS instrument from Malvern on a $0.1 \mathrm{wt} . \%$ CNC suspension using the Smoluchowski approximation.

Carboxylation of Cellulose Nanocrystals by TEMPO Oxidation. Cellulose nanocrystals resulting from sulfuric acid hydrolysis of cotton linters were subjected to TEMPO-mediated oxidation as previously reported. ${ }^{19,42-44} \mathrm{NaBr}(1.588 \mathrm{~g}, 15.4 \mathrm{mmol})$ and TEMPO (135 mg, $0.86 \mathrm{mmol})$ were added to $500 \mathrm{~mL}$ of a $1 \mathrm{wt} . \%$ cellulose nanocrystals suspension $(30.8 \mathrm{mmol}$ anhydroglucose units) and magnetic stirring was applied for $1 \mathrm{~h} . \mathrm{NaOCl}(1.46 \mathrm{M}, 21.1 \mathrm{~mL}$ corresponding to 1 equivalent per anhydroglucose unit) was then added dropwise under stirring to the cellulose suspension. During $\mathrm{NaOCl}$ addition, the $\mathrm{pH}$ was maintained at 10 by using a $0.5 \mathrm{M} \mathrm{NaOH}$ solution. When the $\mathrm{pH}$ value stabilized, the TEMPO reaction was stopped by adding methanol $(20 \mathrm{~mL})$ and the $\mathrm{pH}$ was adjusted to 7 with $0.5 \mathrm{M} \mathrm{HCl}$. The resulting suspensions were washed three times with distilled water by successive centrifugation at $20,000 \mathrm{~g}$ and redispersion. $0.5 \mathrm{M} \mathrm{NaCl}$ was added to facilitate the 
nanoparticle separation and the washing procedure was repeated three times using a $0.1 \mathrm{~N}$ $\mathrm{HCl}$ solution. After dialysis against distilled water, a colloidal suspension stable over years was obtained.

Polymer grafting by peptidic coupling in water. The grafting of amine-terminated Jeffamine ${ }^{\circledR}$ polyetheramines was achieved through peptidic coupling according to Bulpitt and Aeschlimann ${ }^{45}$ and is described in more details in our previous works. ${ }^{19-21}$ The pure polymer was added ( $\mathrm{N}_{\mathrm{p}}$ moles per carboxyl unit measured by conductometry) to a $1 \mathrm{wt} . \%$ carboxylated $\mathrm{CNC}$ suspension and stirred until dissolution. The reaction was performed at $4{ }^{\circ} \mathrm{C}$ to ensure that the macromolecules were under good solvent conditions. The $\mathrm{pH}$ was adjusted to 7.5-8.0 before the addition of $2 \mathrm{~mL}$ of an aqueous solution containing $\mathrm{N}$-(3-dimethylaminopropyl)N'-ethylcarbodiimide hydrochloride (EDAC) and N-hydroxysuccimide (NHS) $\left(\mathrm{N}_{\mathrm{EDAC}}\right.$ and $\mathrm{N}_{\mathrm{NHS}}$ mol per carboxyl group, respectively). The reaction was carried out with $\mathrm{N}_{\mathrm{P}}=\mathrm{N}_{\mathrm{EDAC}}=\mathrm{N}_{\mathrm{NHS}}=4$. The reaction lasted $24 \mathrm{~h}$ at room temperature under stirring while maintaining the $\mathrm{pH}$ of the mixture at 7.5-8.0 using $0.5 \mathrm{M} \mathrm{NaOH}$ or $0.5 \mathrm{M} \mathrm{HCl}$. The $\mathrm{pH}$ was finally lowered to $1-2$ by addition of $0.5 \mathrm{M} \mathrm{HCl}$ and the resulting suspension was dialyzed against distilled water to remove excess reagents including non-grafted polyetheramines. Unless otherwise stated, the $\mathrm{pH}$ of the suspension was about 7.5. As described in our previous work, ${ }^{19}$ the degrees of substitution were measured by conductometric titration and solid-state NMR and values ranging from 0.02 to $0.05 \mathrm{~mol}$ of polymer per mol of anhydroglucose unit were found depending on the reaction conditions.

Small Angle Neutron Scattering (SANS). SANS experiments were performed either at Laboratoire Léon Brillouin (Saclay, France) on the instrument PAXY or at Institut Laue Langevin (Grenoble, France) on the instrument D22. On PAXY or D22, three configurations (average wavelength and detector distance being, respectively, $5 \AA$ at $1 \mathrm{~m}, 5 \AA$ at $5 \mathrm{~m}$ and 
$13 \AA$ at $5 \mathrm{~m}$ ) or two configurations (5.86 $\AA$ at $2 \mathrm{~m}$ and $5.86 \AA$ at $14 \mathrm{~m})$ were used, respectively, to access a momentum transfer range $Q$ from $4.10^{-3} \AA^{-1}$ to $0.4 \AA^{-1}$. The radially averaged spectra were corrected for solvent, empty cell, incoherent scattering and background noise to yield intensity, $I$, in $\mathrm{cm}^{-1} v s$ scattering vector, $Q$, in $\AA^{-1}$ plots. The general expression of the scattering intensity as a function of $Q$ for rigid centrosymmetric objects of homogeneous scattering length density (SLD), $\rho$, is

$$
I(Q)=\Delta \rho^{2} n V^{2} P(Q) S(Q)=\Delta \rho^{2} \phi V P(Q) S(Q)
$$

where $\Delta \rho=\rho-\rho_{s}$ is the difference in SLD between the considered particles and the solvent, $n$ is the number of particles per unit of volume, $V$ is their individual volume, $P(Q)$ is the form factor (related to the shape and size of the particles) and $S(Q)$ is the structure factor (describing the interactions between the particles). With this notation, the volume fraction is $\phi=n V$.

Form factors for SANS data analysis. As-prepared cotton CNCs were shown to be comprised of 2 to 3 laterally aggregated elementary crystallites. ${ }^{46}$ In agreement with these results, dilute suspensions of as-prepared CNCs were fitted using the form factor of a rectangular parallelepiped, averaged over all orientations in space (Eq. 1). For this purpose, a concentration of $1 \mathrm{wt} \%$ was assumed low enough to neglect the interactions between the CNCs and consider that $S(Q)=1$ over the whole $Q$-range (i.e., the system behaves like a perfect gas). It was further recently shown that this model was very well adapted to fit the SANS spectrum of $0.78 \mathrm{wt} . \% \mathrm{CNCs}$ derived from Whatman filters, which is very close to our experimental conditions. ${ }^{23}$

The form factor $P(Q)$ is defined as the squared modulus of the scattering amplitude $F(Q)$. To simplify the notations used throughout this work, we introduce for a simple rectangular 
parallelepiped the volume-weighted form factor amplitude $\bar{F}(Q, \theta, \varphi, H, W, L)=$ $V F(Q, H, W, L, \theta, \varphi)$, defined as ${ }^{47-48}$

$\bar{F}(Q, H, W, L, \theta, \varphi)=(H W L) j_{0}\left(Q_{x} \frac{H}{2}\right) j_{0}\left(Q_{y} \frac{W}{2}\right) j_{0}\left(Q_{z} \frac{L}{2}\right)$

where $H, W$ and $L$ respectively stand for the height, width and length of the CNC rods, $j_{0}(x)=(\sin x) / x$ is the 0th-order spherical Bessel function of the first kind and $Q_{x}=$ $Q \sin \theta \cos \varphi, Q_{y}=Q \sin \theta \sin \varphi$ and $Q_{z}=Q \cos \theta$. The angles $\theta$ and $\varphi$ are the polar and azimuthal angles in spherical coordinates and allow for the orientation average of the volumeweighted form factor over the full solid angle:

$$
\langle\bar{P}(Q)\rangle_{R}=\frac{2}{\pi} \int_{0}^{\pi / 2} \int_{0}^{\pi / 2}|\bar{F}(Q, \theta, \varphi)|^{2} \sin \theta d \theta d \varphi
$$

This latter expression describes the case of anisometric scatterers that are freely rotating in suspension, and, in the following, all integrals for orientation averaging will be shortened for convenience as $\langle\ldots\rangle_{R}$. Combining Equations 1, 2 and 3, the scattering intensity for a parallelepipedic $\mathrm{CNC}$ is then given by $I(Q)=\Delta \rho_{\mathrm{CNC}}^{2} \phi V_{\mathrm{CNC}}^{-1}\langle\bar{P}(Q)\rangle_{R}$, where $V_{\mathrm{CNC}}=$ $H W L$ is the volume of a single CNC.

For polymer-decorated CNCs, first a core-shell rectangular parallelepipedic model was considered ("model 1"), where the core consists of a CNC with a scattering length density $\rho_{\mathrm{CNC}}$ and the shell consists of a polymer layer with a SLD $\rho_{\text {Poly }}$. This model considers the polymer shell as a homogeneous one, with a smooth interface, and therefore does not take into account the polymer conformation. For this first model, the SLD-weighted and volumeweighted scattering amplitude, $\overline{\bar{F}}_{\mathrm{CNC} @ \text { Poly }}^{\text {model1 }}(Q)$, is given by

$$
\overline{\bar{F}}_{\mathrm{CNC} @ \text { Podely }}^{\text {moly }}(Q)=\left(\rho_{\text {Poly }}-\rho_{s}\right) \bar{F}_{\mathrm{T}}(Q)+\left(\rho_{\mathrm{CNC}}-\rho_{\text {Poly }}\right) \bar{F}_{\mathrm{CNC}}(Q)
$$


where $\bar{F}_{\mathrm{CNC}}(Q)=\bar{F}(Q, H, W, L, \theta, \varphi)$ and $\bar{F}_{\mathrm{T}}(Q)=\bar{F}(Q, H+2 h, W+2 h, L+2 h, \theta, \varphi)$ are derived from Equation 2, and $h$ is the thickness of the polyetheramine shell. Finally, the total scattering intensity of this core-shell parallelepiped can be written as:

$$
I_{\mathrm{CNC} @ \text { Poly }}^{\text {model }}(Q)=\phi V_{T}^{-1}\left\langle\left|\overline{\bar{F}}_{\mathrm{CNC} @ \text { Poly }}^{\text {moly }}(Q)\right|^{2}\right\rangle_{R}
$$

where the total scattering volume $V_{T}$ is now including the thickness $h$.

A second model (“model 2") considering parallelepipeds with grafted Gaussian chains was also used. ${ }^{49-50}$ The complete equation for this effective, SLD-weighted and volumeweighted form factor is given by:

$$
\begin{gathered}
\bar{P}_{\mathrm{CNC} @ \text { moly }}^{\text {mole }}(Q)=\Delta \rho_{\mathrm{CNC}}^{2}\left|\bar{F}_{\mathrm{CNC}}(Q)\right|^{2}+ \\
+N_{a}\left(V_{\text {Poly }} \Delta \rho_{\text {Poly }}\right)^{2} \phi\left(Q, R_{g}\right)+ \\
+2 \Delta \rho_{\mathrm{CNC}} \bar{F}_{\mathrm{CNC}}(Q) N_{a} V_{\text {Poly }} \Delta \rho_{\text {Poly }} \psi\left(Q, R_{g}\right) r\left(Q, H+2 d R_{g}, W+2 d R_{g}, L+2 d R_{g}\right)+ \\
+N_{a}\left(N_{a}-1\right)\left(V_{\text {Poly }} \Delta \rho_{\text {Poly }}\right)^{2} \psi^{2}\left(Q, R_{g}\right) Y^{2}\left(Q, H+2 d R_{g}, W+2 d R_{g}, L+2 d R_{g}\right)
\end{gathered}
$$

where the first term on the right hand side accounts for the form factor of the bare CNC (see Eq. 2) and the second term for the contribution of $N_{a}$ polyetheramine chains, by means of the Debye function, $\phi\left(Q, R_{g}\right)$. This function corresponds to the self-correlation term of a Gaussian chain polymer of radius of gyration $R_{g}$ :

$$
\phi\left(Q, R_{g}\right)=2 \frac{e^{-x}-1+x}{x^{2}}, \text { where } x=\left(Q R_{g}\right)^{2}
$$

The third term in Equation 6 is the cross-term between the core $\mathrm{CNC}$ and the polyetheramine chains, and the fourth and last term corresponds to the cross-term among two chains. ${ }^{42-43}$ In these last terms, the function $\psi\left(Q, R_{g}\right)$ represents the equivalent scattering amplitude of the Gaussian chain: 


$$
\psi\left(Q, R_{g}\right)=\frac{1-e^{-x}}{x}, \text { where } x=\left(Q R_{g}\right)^{2}
$$

The parameters $V_{\text {Poly }}$ and $N_{a}$ are, respectively, the volume of a polyetheramine chain block and the number of grafted chains on the surface of the parallelepiped. Here again, we use the notation $\Delta \rho_{i}=\rho_{i}-\rho_{s}$, where $i$ stands for cellulose (CNC) or polyetheramine (Poly). The function $Y(Q, X, Y, Z)$ corresponds to the scattering amplitude of a parallelepiped shell of infinite-thin thickness with dimensions $(X Y Z)$, and $d R_{\mathrm{g}}$ represents the distance between the CNC core surface and the center of mass of the polymer chain. The function $Y(Q, H+$ $2 d R g, W+2 d R g, L+2 d R g$ thus describes the distribution of the grafted chains along the surface of the core. Its expression is given by:

$$
\begin{aligned}
Y(Q, X, Y, Z)= & \frac{Y Z}{Y Z+X Z+X Y} \cos \left(Q_{x} \frac{X}{2}\right) j_{0}\left(Q_{y} \frac{Y}{2}\right) j_{0}\left(Q_{z} \frac{Z}{2}\right) \\
& +\frac{X Z}{Y Z+X Z+X Y} j_{0}\left(Q_{x} \frac{X}{2}\right) \cos \left(Q_{y} \frac{Y}{2}\right) j_{0}\left(Q_{z} \frac{Z}{2}\right) \\
& +\frac{X Y}{Y Z+X Z+X Y} j_{0}\left(Q_{x} \frac{X}{2}\right) j_{0}\left(Q_{y} \frac{Y}{2}\right) \cos \left(Q_{z} \frac{Z}{2}\right)
\end{aligned}
$$

In this second model the polydispersity of the CNC nanorods was also included in order to take into account the lack of defined oscillation in the high $Q$-range. As in this very range the scattering oscillation from a monodisperse parallelepiped would be given by the shortest dimension, $H$, the polydispersity integration was solely applied to this parameter. Then, the final scattering intensity can be written as:

$$
I_{\mathrm{CNC} @ \text { Poly }}^{\text {model2 }}(Q)=n \int\left\langle\overline{\bar{P}}_{\mathrm{CNC} @ \mathrm{moly}}^{\text {mol2 }}(Q)\right\rangle_{R} \mathcal{L}(H, \mu, \sigma) d H
$$

where $\mathcal{L}(H, \mu, \sigma)$ is a log-normal probability distribution function (PDF), whose parameters $\mu$ and $\sigma$ define the mean value and the standard deviation of the natural logarithm of $H$. 


\section{Results}

As shown in our previous work and further evidenced by ${ }^{13} \mathrm{C}$ solid-state NMR and infrared measurements, thermosensitive Jeffamine ${ }^{\circledR}$ polyetheramines can be successfully covalently grafted onto CNCs using the grafting onto strategy through peptide coupling after TEMPO oxidation. ${ }^{19-21}$ When the reaction is performed in water, a degree of substitution (DS) in the 0.03-0.05 range is obtained, depending on the polymer. These DS values correspond to a moderate grafting density, which suggests that these grafted polymer chains of relatively low $M_{w}$ (Table 1) are in a mushroom regime, i.e. without polymer-polymer interactions between vicinal chains. To get more insight into the structure of such polymer-decorated CNCs, SANS experiments were carried out. To facilitate data analysis, the SANS spectra of each individual component were also recorded separately.

\section{Single component characterization}

The SANS study of the initial sulfated CNCs was performed from protonated nanoparticles, which were freeze-dried and redispersed in heavy water at a concentration of $1 \mathrm{wt} . \%$. Using $\mathrm{D}_{2} \mathrm{O}$ as solvent maximizes the constrast $\Delta \rho^{2}$ in Equation 1 , hence optimizing the signal-tonoise ratio of scattered intensity, and minimizing the incoherent scattering contribution generated by $\mathrm{H}_{2} \mathrm{O}$. Prior to freeze-drying, as demonstrated by Beck-Candanedo et al. ${ }^{51}$ the successful redispersion of sulfated CNCs in water requires to exchange the proton counterions (H-NCC acid form) by sodium counterions (neutral salt form Na-NCC), which was carried out by $\mathrm{pH}$ neutralization with sodium hydroxide. The excellent redispersion of the CNCs in $\mathrm{D}_{2} \mathrm{O}$ was attested by dynamic light scattering measurements, whereby the particle size distribution obtained in $\mathrm{D}_{2} \mathrm{O}$ fully superimposed with the initial one measured in $\mathrm{H}_{2} \mathrm{O}$ before freeze-drying (data not shown).

The SANS spectra of a 1 wt.\% cotton CNCs suspension is shown in Figure 1. The variation of the scattered intensity can first be qualitatively analyzed. For as-prepared sulfated CNCs, a 
$Q^{-1}$ decay is observed in the low $Q$-region, showing that CNCs exhibit a rod-like morphology. In the high $Q$-region, where the interface between the particles and the solvent is probed, a $Q^{-4}$ decay is measured, which demonstrates a sharp and smooth interface with the solvent (Porod law). This result is consistent with the well-ordered character of the CNC surface and its low roughness. Given the low concentration of the samples (about five times lower than the onset of formation of the anisotropic phase), the scattered intensity can be considered as resulting solely from the isolated $\mathrm{CNC}$ form factor. Consequently, a quantitative analysis of the SANS spectrum of CNCs was performed using the form factor of isotropically distributed rectangular parallelepipeds given in Equation 1, as used by different groups to extract the dimensions of CNCs. ${ }^{23,27,30,37-38}$

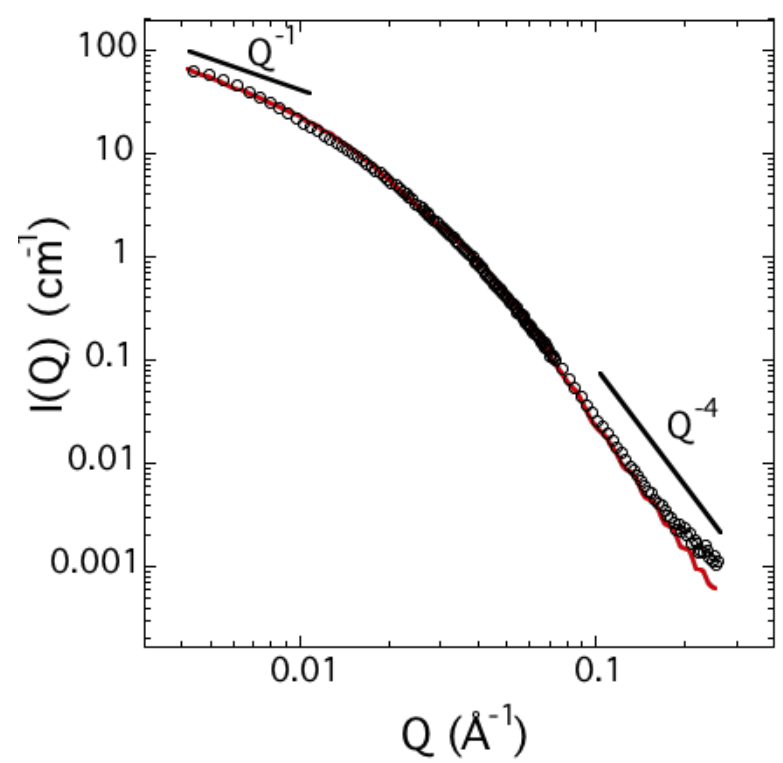

Figure 1. SANS spectrum of a $1 \mathrm{wt} . \%$ as-prepared sulfated CNCs suspension (open circles). The red continuous line is a fit of the data using the form factor of a parallelepiped, while solid straight lines are guides to the eye for the characteristic decay of the intensity in the different $Q$-regions. 
As shown in Figure 1, a very good agreement between the model and the data was achieved, using dimensions of $L=129 \mathrm{~nm}$ for the length, $W=28.6 \mathrm{~nm}$ for the width and $H=4 \mathrm{~nm}$ for the thickness. The high quality of the fit using a pure form factor analysis readily shows the that the structure factor can be considered as equal to unity. The confidence range about the length cannot be accurately extracted due to the lack of data in the low Q range (Guinier plateau) but can be estimated to be equal to a few tens of nanometers. For the width and height, errors on the mean values are respectively equal to $10 \%( \pm 3 \mathrm{~nm})$ and $2 \%(\sim \pm 0.1$ $\mathrm{nm})$. These dimensions are highly consistent with our TEM data and previously reported results. ${ }^{19,46}$ Additionally, a very good agreement is noted with the width and thickness values extracted by Cherhal et al. from SANS data on CNC suspensions obtained from a comparable cellulose source and preparation protocol. ${ }^{23}$ These results confirm that cotton-derived CNCs are composed of few laterally associated elementary crystallites, each of the crystallite having a square-shaped cross-section and length comparable to that of the final particles. Such a composite morphology may reflect the tight organization of the cellulose microfibrils in the initial cotton linters cell wall. ${ }^{23,46}$

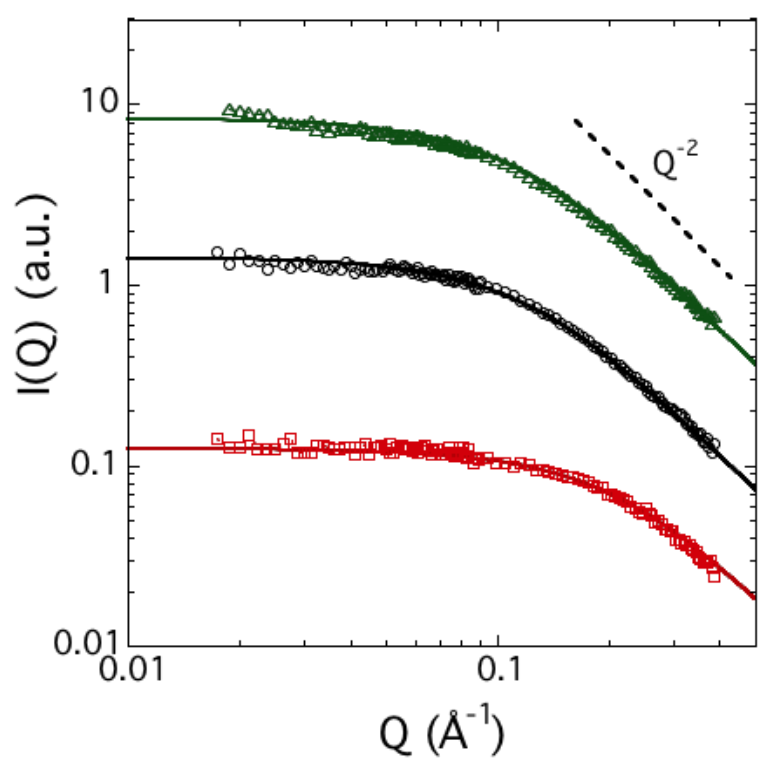


Figure 2. SANS spectra of 2 wt.\% Jeffamine® polyetheramines M600 (red), M2005 (black) and M2070 (blue) solutions in $\mathrm{D}_{2} \mathrm{O}$. Curves have been arbitrarily shifted for clarity. The dotted straight line shows a $Q^{-2}$ decay and continuous lines are fits using the Debye function (Equation 7).

Figure 2 shows the SANS spectra of 2 wt.\% Jeffamine polyetheramine M600, M2005 and M2070 solutions in $\mathrm{D}_{2} \mathrm{O}$. All spectra exhibit the same features, i.e. a $Q^{-2}$ decay at high $Q$ values and a plateau when $Q$ tends to zero, which are typical features of individualized polymer chains in theta solvent. The fit of the experimental curves was performed using the Debye function, $\phi\left(Q, R_{g}\right)$ (Equation 7), for Gaussian polymer chains. ${ }^{52}$ As shown in Figure 2, an excellent agreement is obtained for the three polymer chains investigated, from which $R_{g}$ values of $0.7,1.2$ and $1.3 \mathrm{~nm}$ were obtained for Jeffamine ${ }^{\circledR}$ polyetheramines M600, M2005 and M2070, respectively. These values consistently scale with the molecular weights of the polyetheramines, which are equal to 600,2000 and 2000 g.mol ${ }^{-1}$ for M600, M2005 and M2070 polymers, respectively (Table 1).

Table 1. Characteristics of the Jeffamine ${ }^{\circledR}$ polyetheramines. The radii of gyration are derived from the analysis of the SANS data using the Debye function.

\begin{tabular}{ccccc} 
Polymer & EO/PO ratio & $\operatorname{LCST}\left({ }^{\circ} \mathrm{C}\right)$ & $M_{w}\left(\mathrm{~g} \cdot \mathrm{mol}^{-1}\right)$ & $R_{g}(\mathrm{~nm})$ \\
\hline M600 & $1 / 9$ & 55 & $\sim 600$ & 0.7 \\
M2005 & $10 / 31$ & $\sim 16$ & $\sim 2000$ & 1.2 \\
M2070 & $29 / 6$ & 65 & $\sim 2000$ & 1.3
\end{tabular}

\section{Qualitative analysis of polymer-decorated CNC spectra}


As for as-prepared sulfated CNCs, polyetheramine- $g$-CNC suspensions were freeze-dried and redispersed in $\mathrm{D}_{2} \mathrm{O}$ at a concentration equal to $1 \mathrm{wt} . \%$. As we already reported, ${ }^{20}$ polyetheramine-g-CNCs readily redisperse in water after freeze-drying without special treatment, in contrast to sulfated CNCs. The SANS spectra of a M2005-g-CNC suspension and of the corresponding as-prepared sulfated $\mathrm{CNC}$ suspension were recorded at $8{ }^{\circ} \mathrm{C}$ (Figure 3). This temperature is $8 \mathrm{~K}$ below the LCST of the polymer, which ensures that the chains are in good solvent conditions and that individual polymer-decorated CNCs are probed in absence of any aggregation.

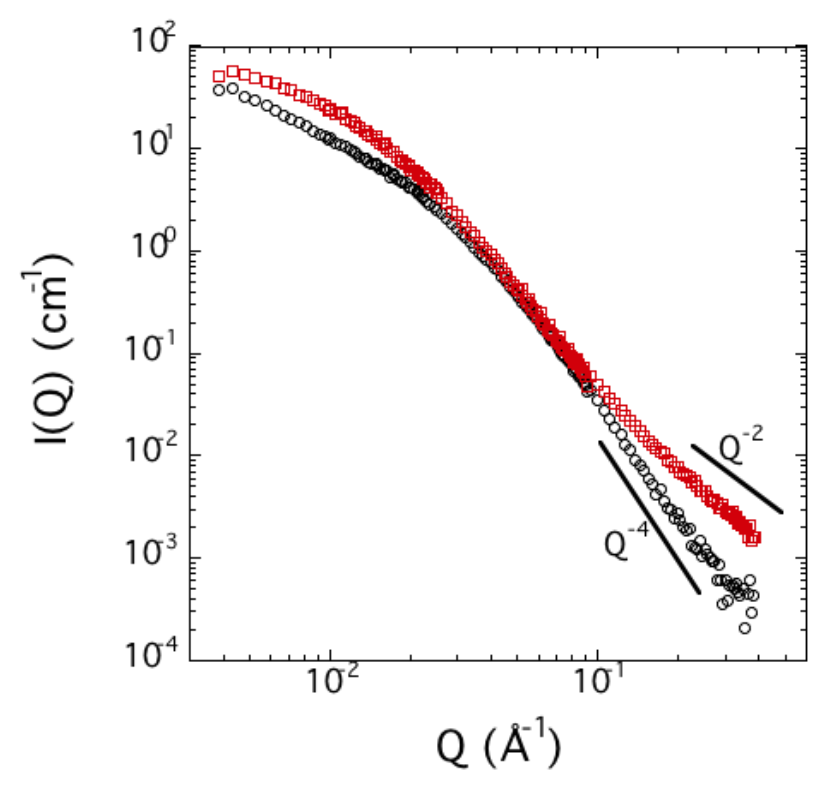

Figure 3. SANS spectra of 1 wt.\% as-prepared sulfated CNCs (black circles) and M2005-gCNCs (red squares) suspensions. The measurements were performed at $8{ }^{\circ} \mathrm{C}$, i.e., $8 \mathrm{~K}$ below the LCST of Jeffamine polyetheramine M2005. Solid straight lines show the characteristic decay of the intensity in the different $Q$-regions.

The spectrum corresponding to M2005-g-CNCs significantly differs from the one of asprepared sulfated CNCs. First, a higher intensity at low $Q$ and a shift towards lower $Q$ (typically from 0.02 to $0.01 \AA^{-1}$ ) of the cross-over regime between the $Q^{-1}$ and $Q^{-2}$ decays 
are observed for M2005-g-CNCs when compared to the parent CNCs. Both features can be attributed to the presence of the polymer chain corona that increases the cross-section (width and thickness) of the nanoparticles. This excess of intensity is not accompanied by a steep rise but tends to plateau for the lowest $Q$-values, as in the case of as-prepared CNCs. This observation is consistent with a non-aggregated nanoparticle state and shows that both samples have a finite cross-section, the one for M2005- $g$-CNCs being slightly larger than the one for sulfated CNCs. As shown in our previous work, for temperatures below the LCST, the presence of the surface chains contributes to colloidal stability due to steric repulsions. A remarkable difference can also be observed in the high $Q$-region, where the interface between the nanoparticles surface and the solvent is probed. Unlike the $Q^{-4}$ decay observed for CNCs, which is typical of a sharp interface with a low roughness in agreement with the crystalline CNC-water smooth interface, a $Q^{-2}$ decay is obtained for M2005-g-CNCs at this local length scale. The latter behavior is in agreement with the M2005-g-CNC/water interface being composed of polymer chains in theta solvent (Gaussian statistics). Indeed, at this local length the polymer conformation is probed and a $Q^{-1 / v}$ decay is expected for chains displaying a $\square$ Flory exponent $v=0.5$. The overall scattering of the grafted objects in this $Q$-range is dominated by the polymer scattering as its contribution to the scattered intensity is much larger than the one of the CNC core $\left(Q^{-2}\right.$ versus $Q^{-4}$ decay). In the intermediate $Q$-region, the intensity of the grafted sample is very slightly higher than that of the non-grafted one. Overall, all the features displayed by the M2005- $g$-CNC sample are fully consistent with the presence of a polymer corona around the CNCs with chains obeying Gaussian statistics. As shown in Supporting Information Figure S1, the same conclusions can be drawn from spectra recorded for M2070- $g$-CNCs and M600- $g$-CNC. The presence of a polymer corona around the CNCs after grafting of polyetheramines M600, M2005 and M2070 is therefore qualitatively attested by SANS. 


\section{Quantitative modeling of polymer-decorated CNC spectra}

In addition to the qualitative analysis of the SANS data, an alternative fitting approach was carried out. First, data were fitted using the form-factor of core-shell rectangular parallelepipeds with homogeneous but different SLDs for the core (equal to the one of crystalline cellulose) and the shell (representing a homogeneous swollen polymer corona), averaged over all orientations (see experimental section). As shown in Figure 4, a good agreement between the model and the experimental data is obtained at the low and intermediate $Q$-range, up to $Q=0.15 \AA^{-1}$ for M600- $g$-CNC and M2070- $g$-CNC and up to $Q=$ $0.06 \AA^{-1}$ for M2005-g-CNC.

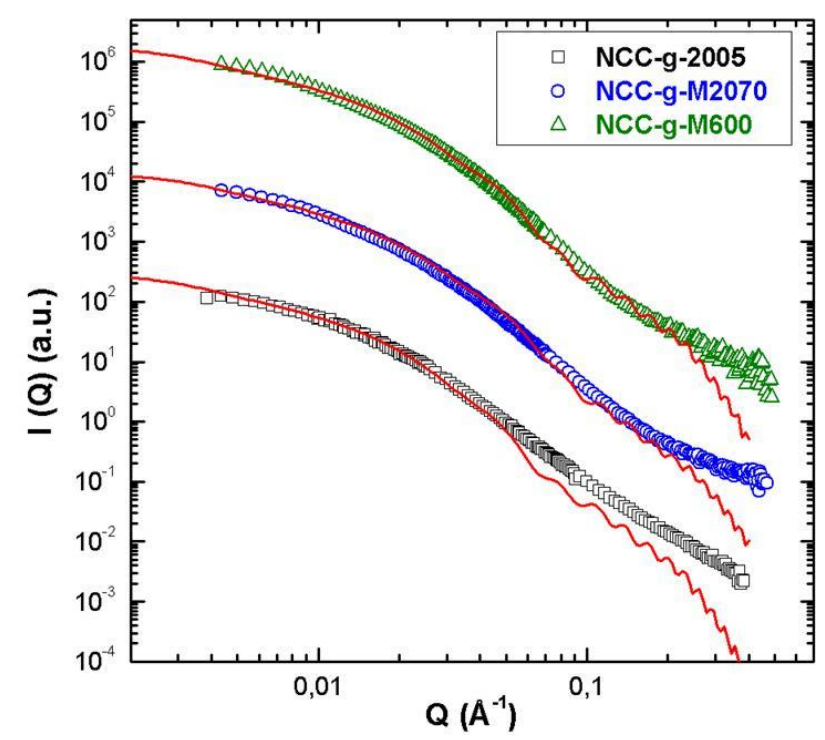

Figure 4. SANS spectra of $10 \mathrm{~g} / \mathrm{L}$ M2005-g-CNCs (black squares), M2070-g-CNCs (blue circles) and M600-g-CNCs (green triangles) suspension in $\mathrm{D}_{2} \mathrm{O}$ at $8{ }^{\circ} \mathrm{C}$. Red lines are best fits using the "model 1" of a simple core-shell parallelepiped (Equation 5).

The poor agreement in the high- $Q$ region is inherent to the model, which considers a homogeneous polymer shell rather than a polymer conformation-dependent SLD variation from the surface. For the three suspensions, the best agreement was obtained for a polymer 
shell thickness, $h$, equal to the radius of gyration of the considered free polymer (Table 1 ). Indeed, when $h>R_{g}$, a discrepancy between this model and the experimental data appears in the high $Q$ region from $0.08 \AA^{-1}$. For $h<R_{g}$, the discrepancy is rather observed in the intermediate or small $Q$ region (between 0.004 and $0.04 \AA^{-1}$ ) (Supporting Information Figure S2).

A more realistic model considering parallelepipeds with surface Gaussian chains was then tested, as described in the experimental section. Aforementioned values of $L=129 \mathrm{~nm}, W=$ $28.6 \mathrm{~nm}$ and the mean value $H=4 \mathrm{~nm}$ (polydispersity index 0.42 ) of the $\mathrm{CNC}$ core, derived from the fitting of as-prepared sulfated CNCs with Equations 1 and 3, were injected as fixed values in the model, and SANS data for dilute suspensions of polyetheramine-g-CNC were fitted using the expression in Equation 10. The polydispersity average was applied by using a log-normal probability distribution function over the shortest side $H$, in agreement with previous work. ${ }^{46}$ SLDs of polyetheramines M600, M2005 and M2070 were calculated as $\rho=$ $0.308 \cdot 10^{-4}, 0.359 \cdot 10^{-4}$ and $0.382 \cdot 10^{-4} \mathrm{~nm}^{-2}$, respectively. As shown in Figure 5 and in contrast to Figure 4, a good agreement between the fit and experimental data could be achieved over the entire Q-range probed for all the three samples. As expected, this result shows that the outer polyetheramine shell is much better described by regular polymer chains with a Gaussian conformation rather than by a homogeneous and smooth corona. The radius of gyration of the grafted chains, the number of chains, $N_{a}$, and the grafting density, $N_{d}$ /area, were extracted from the fits and the results are summarized in Table 2. In the case of polyetheramines M2005 and M2070, a very good agreement between $R_{g}$ in solution (Table 1) and $R_{g}$ at the CNC surface can be noted (respectively 1.2 and $1.3 \mathrm{~nm}$ for Jeffamine® polyetheramine M2005 and 1.3 and $1.2 \mathrm{~nm}$ for Jeffamine® polyetheramine M2070). This result suggests that polymer chains grafted on the surface of CNCs adopt a statistical coil 
conformation. In other words, the grafting density is such that polyetheramines chains on the surface are in a mushroom regime, whereby the chains do not interact with each other. This result is not surprising given the relatively low degrees of substitution of 0.02 measured by solid-state NMR, which corresponds to grafting densities of about 0.2 chain $/ \mathrm{nm}^{2}$, in fair agreement with the values extracted from the fitting of the SANS data ( $N_{d}$ /area values in Table 2). Durand-Gasselin et al. had obtained a polymer layer thickness varying between 2 and $6 \mathrm{~nm}$ by grafting Jeffamine ${ }^{\circledR}$ polyetheramines M600 and M1000 on gold nanoparticles of 2 and $18 \mathrm{~nm}$ in diameter. ${ }^{53}$ These values are significantly higher than ours for M600-g-CNC. This can be simply attributed to a higher grafting density (of $\sim 4$ chain/nm ${ }^{2}$ obtained for the grafting of M600 on gold nanoparticles, which corresponds to a mean distance of $0.7 \mathrm{~nm}$ only, lower that $R_{g}$ ), which forces the chains to adopt a stretched conformation and results in a polymer brush regime.

Some discrepancy is observed in the case of Jeffamine ${ }^{\circledR}$ polyetheramine $\operatorname{M600}\left(R_{g}=0.7 \mathrm{~nm}\right.$ in solution and $1.1 \mathrm{~nm}$ at the surface), which can be attributed to a lack of accuracy due to the small size of the chain and/or can be ascribed to strong correlations between $R_{g}, N_{a}$, and $V_{\text {Poly }}$ values. 


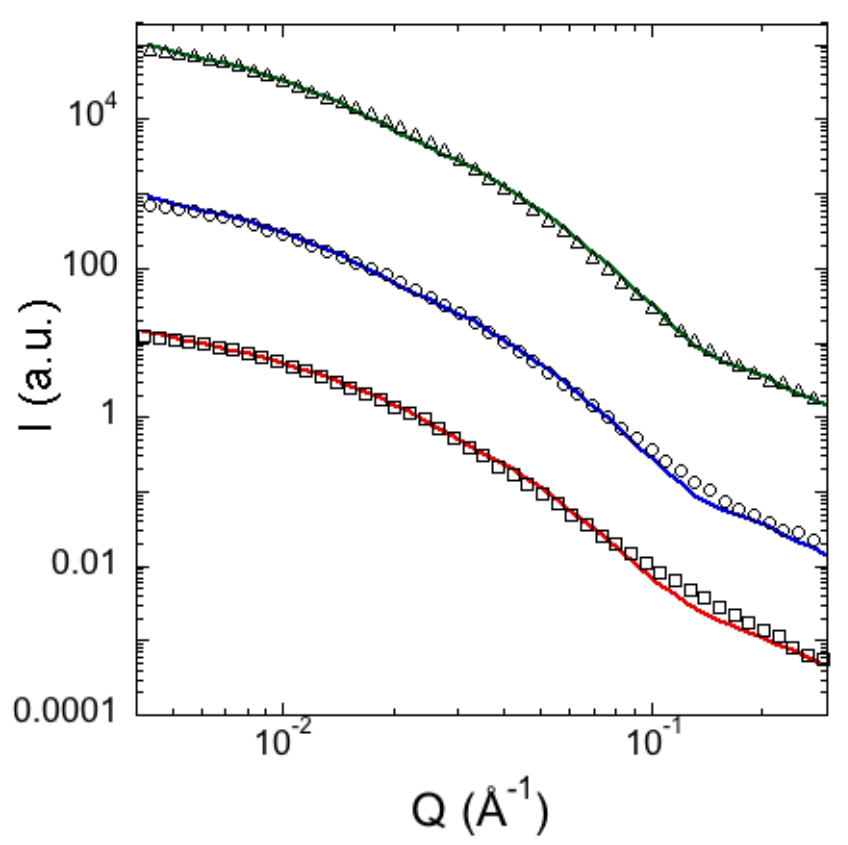

Figure 5. SANS spectra of 1 wt.\% M2005- $g$-CNC (black squares), M2070- $g$-CNC (blue circles) and $\mathrm{M} 600-\mathrm{g}-\mathrm{CNC}$ (green triangles) suspensions in $\mathrm{D}_{2} \mathrm{O}$ at $8{ }^{\circ} \mathrm{C}$. Continuous lines are best fits using the "model 2" based on a core parallelepiped and a shell of Gaussian chains (Equation 10). Spectra and fits have been shifted vertically for clarity.

Table 2. Parallelepiped with Gaussian chains form factor analysis of polyetheramine- $g$-CNCs

\begin{tabular}{ccccc} 
Sample & $R_{g}(\mathrm{~nm})$ & $N_{a}$ & $N_{a} /$ area & $V_{\text {Poly }}\left(\mathrm{nm}^{3}\right)$ \\
& & & $\left(\mathrm{nm}^{-2}\right)$ & \\
\hline M600-g-CNC & 1.15 & 890 & 0.1 & 1.8 \\
M2005-g-CNC & 1.32 & 720 & 0.09 & 2.9 \\
M2070-g-CNC & 1.23 & 670 & 0.08 & 2.9
\end{tabular}

\section{Probing the effect of temperature using SANS}

As shown in our previous work, the grafting of Jeffamine ${ }^{\circledR}$ polyetheramine M2005 onto the surface of $\mathrm{CNCs}$ leads to a reversible temperature-induced aggregation behavior when the samples are heated above the LCST of the polymer, equal to $16^{\circ} \mathrm{C} .{ }^{19-20} \mathrm{~A}$ remarkable 
reversibility and reproducibility of the phenomenon was evidenced by DLS measurements, while the aggregate morphology could be observed by TEM and cryo-TEM. The latter observations had shown that below the LCST the polymer-decorated CNCs consisted in stable individualized rod-like nanoparticles that looked similar to the initial sulfated CNCs since the polymer shell was not dense enough to generate any detectable contrast in the TEM images. As aforementioned, SANS allowed us not only to confirm the colloidal stability below the LCST and the presence of individual particles in the suspension, but to evidence the presence of a polymer shell at the surface of the CNCs. In addition to negative surface charges, these polymer chains contribute to the colloidal stability by providing repulsive steric interactions when the temperature is below the LCST. For samples prepared at $\mathrm{T}=40{ }^{\circ} \mathrm{C}$ (i.e., $24 \mathrm{~K}$ above the LCST), TEM images shown in our earlier work had revealed aggregates with a length ranging from 300 to $700 \mathrm{~nm}$, and a width from 75 to $200 \mathrm{~nm} \cdot{ }^{21}$ Given the average dimensions of the initial CNCs, these aggregates likely result from the lateral assembling of M2005-gCNCs into bundles. Their apparent width corresponds approximately to that of 4 to 10 individual particles, while their length is 2 to 4 times that of initial CNCs. This temperatureinduced aggregation was further investigated by SANS since, unlike DLS and TEM, this technique allows for probing concentrated suspensions and the observation of structural changes. Figure 6 shows the SANS spectra of a 1 wt. $\%$ M2005- $g$-CNCs suspension in $\mathrm{D}_{2} \mathrm{O}$ at $8,15,25$ and $40^{\circ} \mathrm{C}$. As the temperature is progressively increased from $8^{\circ} \mathrm{C}$ (below the LCST) to $15^{\circ} \mathrm{C}$ (close to the LCST) and finally to 25 and $40^{\circ} \mathrm{C}$ (above the LCST), an increase in the scattered intensity at small $Q$ values is clearly observed, reflecting the aggregation of M2005-g-CNCs. Although a limited scattering vector range was probed, the observed increase in intensity appears moderate and seems to plateau, suggesting the formed aggregates are of finite size. These observations are in agreement with those from TEM and DLS. ${ }^{19-20}$ The increase of the intensity in the low- $Q$ region with the temperature is also 
accompanied by a variation of the slope in the high- $Q$ region, reflecting variations in the particle-solvent interface with the increase of the temperature. Indeed, at $T<$ LCST, the behavior at high- $Q$ is close to a $Q^{-2}$ decay, which is consistent with the Gaussian coil conformation signature (theta solvent) of the polymer chains in the outer region of M2005-gCNCs. In contrast, at $\mathrm{T}=25^{\circ} \mathrm{C}$, less than $10 \mathrm{~K}$ above the LCST, a decay close to $Q^{-3}$ is observed and at $40{ }^{\circ} \mathrm{C}, 24 \mathrm{~K}$ above the LCST, it tends to $Q^{-4}$. This behavior can be attributed to the progressive desolvation of the surface polymer shell. At the highest temperature probed, almost all water molecules are expelled and collapsed chains form a smooth layer at the CNC surface, leading to a $Q^{-4}$ Porod characteristic scattering decay. Closer to the LCST, the polyetheramine chains are not fully desolvated and form a heterogeneous and rough layer, yielding the $Q^{-3}$ decay expected for rough surfaces.

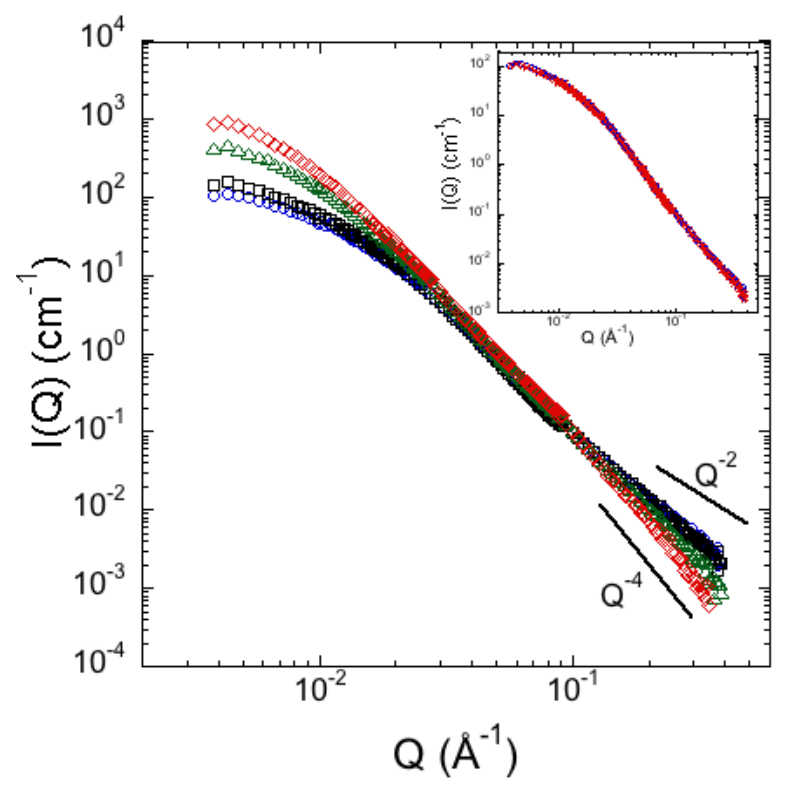

Figure 6. SANS spectra of a $10 \mathrm{~g} / \mathrm{L}$ M2005- $g$-CNCs suspension in $\mathrm{D}_{2} \mathrm{O}$ at $8{ }^{\circ} \mathrm{C}$ (blue circles), $15^{\circ} \mathrm{C}$ (black squares), $25^{\circ} \mathrm{C}$ (green triangles) and $40{ }^{\circ} \mathrm{C}$ (red diamonds). Solid straight lines show the characteristic decay of the intensity in the high $Q$-region. Inset: SANS spectra of the 
same suspension in $\mathrm{D}_{2} \mathrm{O}$ at $8{ }^{\circ} \mathrm{C}$ before any thermal treatment (blue circles) and after heating at $40^{\circ} \mathrm{C}$ and subsequent cooling at $8{ }^{\circ} \mathrm{C}$ (red crosses).

These detailed information extracted from SANS measurements are perfectly in line with previously reported TEM and DLS data and support the following interaction variation mechanism: for temperatures below the LCST of Jeffamine ${ }^{\circledR}$ polyetheramine M2005 (e.g., $8{ }^{\circ} \mathrm{C}$ in Figure 3), grafted polymer chains are in theta solvent conditions and behave as Gaussian coils that generate steric repulsion forces between M2005-g-CNCs. As a result, individual particles are observed by SANS. It has however to be noted that in the ionic strength conditions probed, these electrostatic repulsions are not strong enough to generate a detectable scattering peak. For temperatures higher than the LCST (e.g., 25 and $40{ }^{\circ} \mathrm{C}$ in Figure 6), polyetheramine chains undergo a collapse since water is then a poor solvent and aggregation based on attractive interactions between hydrophobic polymer globules at the surface of CNCs take place. As previously reported, the aggregate size strongly depends on the presence of remaining electrical charges on the surface of the CNCs. The suppression of these charges by $\mathrm{pH}$ decrease or their screening by salt addition result in bigger size aggregates. ${ }^{20}$ Consistently, SANS data recorded here in conditions where some electrical charges are still present (neutral $\mathrm{pH}$ ) and not screened (no added salt) reveal the presence of finite-size aggregates that are most probably stabilized by electrostatic repulsions. The size of theses aggregates made the samples turbid but no sedimentation could be observed.

The inset in Figure 6 shows a perfect superposition of the SANS spectra of a M2005-g-CNCs suspension originally at $8{ }^{\circ} \mathrm{C}$ and the one recorded on the same suspension after it was successively heated to $40{ }^{\circ} \mathrm{C}$ and cooled down to $8{ }^{\circ} \mathrm{C}$. This comparison confirms a perfect reversibility of the aggregation phenomenon at all probed lengthscales. 
To probe the effect of concentration on the aggregation process, SANS spectra of M2005-g$\mathrm{CNCs}$ at 1 and 6 wt.\% were measured at 8, 20, 40 and $60{ }^{\circ} \mathrm{C}$. As shown in Supporting Information Figure $\mathrm{S} 3$, the increase in intensity at low- $Q$ is enhanced when the concentration is higher, suggesting that larger size aggregates are formed at $6 \mathrm{wt} . \%$ than at $1 \mathrm{wt} . \%$. The aggregation number could be tentatively derived from the SANS data using the relation $N_{\text {agg }}=S(Q \rightarrow 0)$, where $S(Q)$ is the structure factor. However here the structure factor cannot be rigorously extracted from the data since the form factor is temperature dependent. Consequently, in an attempt to semi-quantitatively describe the results, an apparent aggregation number, $N_{\text {agg }}^{\text {app }}$, was estimated from $N_{\text {agg }}^{\text {app }}=\frac{I_{C, T \neq 8}(Q \rightarrow 0)}{I_{C, T=8}(Q \rightarrow 0)}$.

As shown in Figure 7, aggregates with apparent aggregation numbers between about 4 and 40 are formed. The higher the temperature and/or the concentration, the higher the apparent aggregation number. An apparent aggregation number about 3 times higher is obtained when the concentration is increased from 1 to $6 \mathrm{wt} . \%$ and an about 4 times-increase is observed when the temperature is increased from 20 to $60{ }^{\circ} \mathrm{C}$.

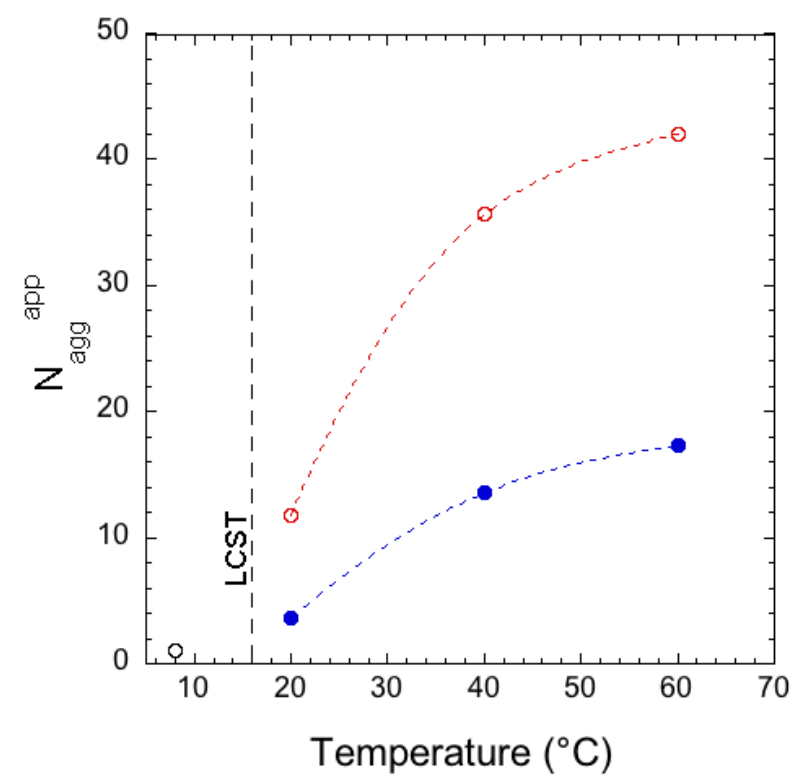


Figure 7. Apparent aggregation number as a function of temperature for M2005-g-CNCs suspensions at $1 \mathrm{wt} . \%$ (full blue circles) and $6 \mathrm{wt} . \%$ (open red circles). The black open circle below the LCST stands for the reference of individual polymer-decorated CNC $\left(N_{\mathrm{agg}}^{\mathrm{app}}=1\right)$. The dashed horizontal line shows the $\operatorname{LCST}\left(16^{\circ} \mathrm{C}\right)$. Blue and red dashed lines are guides to the eye.

\section{Conclusion}

In this work, the potential of SANS to describe the structural features of thermosensitive polymer-decorated CNCs was investigated. In contrast with electron microscopy or AFM observations, the qualitative interpretation of characteristic decays and quantitative analysis with good fitting results of SANS spectra allowed us to extract detailed structural information. At temperatures below the LCST of the thermo-sensitive polymer, grafted polyetheramine chains obey Gaussian statistics and the thickness of the polymer shell around the CNCs is very comparable with the radius of gyration of free chains in aqueous medium. A relatively low grafting density was measured, evidencing that the grafted chains on the surface are in a mushroom regime and not as dense brushes. Despite this low grafting density, the polymer shell ensures an entropic stabilization of the derivatized rods, which results in the enhanced colloidal properties we revealed in previous works. When the temperature of the suspension was increased above the LCST of the thermosensitive polymer, SANS spectra showed the formation of aggregates with an apparent aggregation number that increases both with the sample concentration and temperature deviation from the LCST. When the sample was cooled down below the LCST, SANS evidences a perfect thermo-reversibility at all length-scales, i.e. a complete disintegration of the aggregates into individual particles and the recovery of surface chains in theta solvent. Results obtained here based on SANS measurements are 
helpful in the understanding of the structure-properties relationship of derivatized CNC samples, which in turn broadens their applicative potential.

\section{ASSOCIATED CONTENT}

\section{Supporting Information}

SANS spectra of 1 wt.\% as-prepared CNC, M2070-g-CNCs and M600-g-CNCs suspensions at $8{ }^{\circ} \mathrm{C}(<<$ LCST $)$. Fits using the "model 1 " based on smooth core- shell parallelepipeds. Temperature evolution of $10 \mathrm{~g} / \mathrm{L}$ and $60 \mathrm{~g} / \mathrm{L} \mathrm{M} 2005-\mathrm{g}-\mathrm{CNC}$ suspensions in the $8-60{ }^{\circ} \mathrm{C}$ range.

\section{AUTHOR INFORMATION}

Corresponding Author

*E-mail: bruno.jean@ cermav.cnrs.fr

Present Addresses

${ }^{1}$ F.A.: School of Health Sciences, Modern University for Business and Science, Lebanon

${ }^{2}$ B.F.-P.: Melville laboratory for Polymer Synthesis, Department of Chemistry, University of Cambridge, Lensfield Road, Cambridge CB2 1EW, UK.

${ }^{3}$ E.F.S.: University of Graz, Institute of Molecular Biosciences, Biophysics Division, NAWI Graz, 8010 Graz, Austria and BioTechMed Graz, 8010 Graz, Austria

\section{Financial Support}

Financial support from Institut Carnot PolyNat (ANR N ${ }^{\circ}$ 16-CARN-025-01).

\section{ACKNOWLEDGMENTS}


BJ thanks Laboratoire Léon Brillouin and Institut Laue-Langevin for beamtime allocation.

Huntsman Corporation is acknowledged for the generous gift of Jeffamine ${ }^{\circledR}$ polyetheramine samples. BJ acknowledges the dedication of Orphée reactor and Laboratoire Léon Brillouin staff for providing access in the best possible conditions to neutron scattering instruments till 2019. This article is dedicated to the memory of Isabelle Grillo, who acted as a local contact for some of the SANS experiments presented here.

\section{References}

1. Habibi, Y.; Lucia, L. A.; Rojas, O. J. Cellulose Nanocrystals: Chemistry, SelfAssembly, and Applications. Chem. Rev. 2010, 110, 3479-3500.

2. Klemm, D.; Kramer, F.; Moritz, S.; Lindstrom, T.; Ankerfors, M.; Gray, D.; Dorris, A. Nanocelluloses: A New Family of Nature-Based Materials. Angewandte Chemie-International Edition 2011, 50, 5438-5466.

3. Reid, M. S.; Villalobos, M.; Cranston, E. D. Benchmarking cellulose nanocrystals: from the laboratory to industrial production. Langmuir 2016, 33, 1583-1598.

4. Moon, R. J.; Martini, A.; Nairn, J.; Simonsen, J.; Youngblood, J. Cellulose nanomaterials review: structure, properties and nanocomposites. Chem. Soc. Rev. 2011, 40, 3941-3994.

5. Salas, C.; Nypeloe, T.; Rodriguez-Abreu, C.; Carrillo, C.; Rojas, O. J. Nanocellulose properties and applications in colloids and interfaces. Current Opinion in Colloid \& Interface Science 2014, 19, 383-396.

6. $\quad$ Lagerwall, J. P. F.; Schütz, C.; Salajkova, M.; Noh, J.; Park, J. H.; Scalia, G.; Bergstrom, L. Cellulose nanocrystal-based materials: from liquid crystal self-assembly and glass formation to multifunctional thin films. Npg Asia Materials 2014, 6, e80.

7. Frka-Petesic, B.; Jean, B.; Heux, L. First experimental evidence of a giant permanent electric-dipole moment in cellulose nanocrystals. EPL 2014, 107, 28006.

8. $\quad$ Frka-Petesic, B.; Sugiyama, J.; Kimura, S.; Chanzy, H.; Maret, G. Negative diamagnetic anisotropy and birefringence of cellulose nanocrystals. Macromolecules 2015, 48, 8844-8857.

9. Frka-Petesic, B.; Radavidson, H.; Jean, B.; Heux, L. Dynamically controlled iridescence of cholesteric cellulose nanocrystal suspensions using electric fields. Adv. Mater. 2017, 29, 1606208.

10. Parker, R. M.; Guidetti, G.; Williams, C. A.; Zhao, T.; Narkevicius, A.; Vignolini, S.; Frka-Petesic, B. The Self-Assembly of Cellulose Nanocrystals: Hierarchical Design of Visual Appearance. Adv. Mater. 2018, 30, 1704477.

11. Frka-Petesic, B.; Guidetti, G.; Kamita, G.; Vignolini, S. Controlling the photonic properties of cholesteric cellulose nanocrystal films with magnets. Adv. Mater. 2017, 29, 1701469.

12. Kalashnikova, I.; Bizot, H.; Cathala, B.; Capron, I. New Pickering Emulsions Stabilized by Bacterial Cellulose Nanocrystals. Langmuir 2011, 27, 7471-7479.

13. Kalashnikova, I.; Bizot, H.; Cathala, B.; Capron, I. Modulation of Cellulose Nanocrystals Amphiphilic Properties to Stabilize Oil/Water Interface. Biomacromolecules 2011, 13, 267-275. 
14. Klemm, D.; Cranston, E. D.; Fischer, D.; Gama, M.; Kedzior, S. A.; Kralisch, D.; Kramer, F.; Kondo, T.; Lindström, T.; Nietzsche, S. Nanocellulose as a natural source for groundbreaking applications in materials science: Today's state. Mater. Today 2018, 21, 720748.

15. Eyley, S.; Thielemans, W. Surface modification of cellulose nanocrystals. Nanoscale 2014, 6, 7764-7779.

16. Habibi, Y. Key advances in the chemical modification of nanocelluloses. Chem. Soc. Rev. 2014, 43, 1519-1542.

17. Tang, J.; Sisler, J.; Grishkewich, N.; Tam, K. C. Functionalization of cellulose nanocrystals for advanced applications. J. Colloid Interface Sci. 2017, 494, 397-409.

18. Wohlhauser, S.; Delepierre, G.; Labet, M.; Morandi, G. 1.; Thielemans, W.; Weder, C.; Zoppe, J. O. Grafting Polymers from Cellulose Nanocrystals: Synthesis, Properties, and Applications. Macromolecules 2018.

19. Azzam, F.; Heux, L.; Putaux, J.-L.; Jean, B. Preparation By Grafting Onto, Characterization, and Properties of Thermally Responsive Polymer-Decorated Cellulose Nanocrystals. Biomacromolecules 2010, 11, 3652-3659.

20. Azzam, F.; Siqueira, E.; Fort, S.; Hassaini, R.; Pignon, F.; Travelet, C.; Putaux, J.-L.; Jean, B. Tunable aggregation and gelation of thermoresponsive suspensions of polymergrafted cellulose nanocrystals. Biomacromolecules 2016, 17, 2112-2119.

21. Azzam, F.; Heux, L.; Jean, B. Adjustment of the Chiral Nematic Phase Properties of Cellulose Nanocrystals by Polymer Grafting. Langmuir 2016, 32, 4305-4312.

22. Foster, E. J.; Moon, R. J.; Agarwal, U. P.; Bortner, M. J.; Bras, J.; Camarero-Espinosa, S.; Chan, K. J.; Clift, M. J.; Cranston, E. D.; Eichhorn, S. J. Current characterization methods for cellulose nanomaterials. Chemical Society Reviews 2018, 47, 2609-2679.

23. Cherhal, F.; Cousin, F.; Capron, I. Influence of Charge Density and Ionic Strength on the Aggregation Process of Cellulose Nanocrystals in Aqueous Suspension, as Revealed by Small-Angle Neutron Scattering. Langmuir 2015, 31, 5596-5602.

24. De France, K. J.; Yager, K. G.; Hoare, T.; Cranston, E. D. Cooperative ordering and kinetics of cellulose nanocrystal alignment in a magnetic field. Langmuir 2016, 32, 75647571.

25. Ehmann, H. M. A.; Spirk, S.; Doliska, A.; Mohan, T.; Gossler, W.; Ribitsch, V.; Sfiligoj-Smole, M.; Stana-Kleinschek, K. Generalized Indirect Fourier Transformation as a Valuable Tool for the Structural Characterization of Aqueous Nanocrystalline Cellulose Suspensions by Small Angle X-ray Scattering. Langmuir 2013, 29, 3740-3748.

26. Haywood, A. D.; Weigandt, K. M.; Saha, P.; Noor, M.; Green, M. J.; Davis, V. A. New insights into the flow and microstructural relaxation behavior of biphasic cellulose nanocrystal dispersions from RheoSANS. Soft Matter 2017, 13, 8451-8462.

27. Mao, Y.; Liu, K.; Zhan, C.; Geng, L.; Chu, B.; Hsiao, B. S. Characterization of Nanocellulose Using Small-Angle Neutron, X-ray, and Dynamic Light Scattering Techniques. The Journal of Physical Chemistry B 2017, 121, 1340-1351.

28. Orts, W. J.; Godbout, L.; Marchessault, R. H.; Revol, J. F. Enhanced ordering of liquid crystalline suspensions of cellulose microfibrils: A small angle neutron scattering study. Macromolecules 1998, 31, 5717-5725.

29. Schutz, C.; Agthe, M.; Fall, A. B.; Gordeyeva, K.; Guccini, V.; Salajkova, M.; Plivelic, T. S.; Lagerwall, J. P. F.; Salazar-Alvarez, G.; Bergstroem, L. Rod Packing in Chiral Nematic Cellulose Nanocrystal Dispersions Studied by Small-Angle X-ray Scattering and Laser Diffraction. Langmuir 2015, 31, 6507-6513.

30. Uhlig, M.; Fall, A.; Wellert, S.; Lehmann, M.; Prévost, S.; Wågberg, L.; von Klitzing, R.; Nyström, G. Two-Dimensional Aggregation and Semidilute Ordering in Cellulose Nanocrystals. Langmuir 2016, 32, 442-450. 
31. Tanaka, R.; Kuribayashi, T.; Ogawa, Y.; Saito, T.; Isogai, A.; Nishiyama, Y. Ensemble evaluation of polydisperse nanocellulose dimensions: rheology, electron microscopy, X-ray scattering and turbidimetry. Cellulose 2017, 24, 3231-3242.

32. Liu, Y.; Agthe, M.; Salajková, M.; Gordeyeva, K.; Guccini, V.; Fall, A.; SalazarAlvarez, G.; Schütz, C.; Bergström, L. Assembly of cellulose nanocrystals in a levitating drop probed by time-resolved small angle X-ray scattering. Nanoscale 2018, 10, 18113-18118. 33. Cohen, N.; Ochbaum, G.; Levi-Kalisman, Y.; Bitton, R.; Yerushalmi-Rozen, R. Polymer-induced modification of cellulose nanocrystals assembly in aqueous suspensions. ACS Applied Polymer Materials 2019.

34. Rosén, T.; Wang, R.; Zhan, C.; He, H.; Chodankar, S.; Hsiao, B. S. Cellulose nanofibrils and nanocrystals in confined flow: Single-particle dynamics to collective alignment revealed through scanning small-angle $\mathrm{x}$-ray scattering and numerical simulations. Physical Review E 2020, 101, 032610.

35. Semeraro, E. F.; Hengl, N.; Karrouch, M.; Michot, L. J.; Paineau, E.; Jean, B.; Putaux, J.-L.; Lancelon-Pin, C.; Sharpnack, L.; Pignon, F. Layered organization of anisometric cellulose nanocrystals and beidellite clay particles accumulated near the membrane surface during cross-flow ultrafiltration: In situ SAXS and ex situ SEM/WAXD characterization. Colloids and Surfaces A: Physicochemical Engineering Aspects 2020, 584, 124030.

36. Gicquel, E.; Bras, J.; Rey, C.; Putaux, J.-L.; Pignon, F.; Jean, B.; Martin, C. J. C. Impact of sonication on the rheological and colloidal properties of highly concentrated cellulose nanocrystal suspensions. Cellulose 2019, 1-16.

37. Terech, P.; Chazeau, L.; Cavaille, J. Y. A Small-Angle Scattering Study of Cellulose Whiskers in Aqueous Suspensions. Macromolecules 1999, 32, 1872-1875.

38. Bonini, C.; Heux, L.; Cavaillé, J.-Y.; Lindner, P.; Dewhurst, C.; Terech, P. Rodlike Cellulose Whiskers Coated with Surfactant: A Small-Angle Neutron Scattering Characterization. Langmuir 2002, 18, 3311-3314.

39. Lin, F.; Cousin, F.; Putaux, J.-L.; Jean, B. Temperature-Controlled Star-Shaped Cellulose Nanocrystal Assemblies Resulting from Asymmetric Polymer Grafting. ACS Macro Letters 2019, 345-351.

40. Leguy, J.; Diallo, A.; Putaux, J.-L.; Nishiyama, Y.; Heux, L.; Jean, B. Periodate Oxidation Followed by NaBH4 Reduction Converts Microfibrillated Cellulose into Sterically Stabilized Neutral Cellulose Nanocrystal Suspensions. Langmuir 2018, 34, 11066-11075. 41. Revol, J. F.; Bradford, H.; Giasson, J.; Marchessault, R. H.; Gray, D. G. Helicoidal self-ordering of cellulose microfibrils in aqueous suspension. Int. J. Biol. Macromol. 1992, $14,170-172$.

42. Denooy, A. E. J.; Besemer, A. C.; Vanbekkum, H. Highly selective tempo mediated oxidation of primary alcohol groups in polysaccharides. Recueil Des Travaux Chimiques Des Pays-Bas-Journal of the Royal Netherlands Chemical Society 1994, 113, 165-166.

43. Habibi, Y.; Chanzy, H.; Vignon, M. R. TEMPO-mediated surface oxidation of cellulose whiskers. Cellulose (London) 2006, 13, 679.

44. Saito, T.; Isogai, A. TEMPO-mediated oxidation of native cellulose. The effect of oxidation conditions on chemical and crystal structures of the water-insoluble fractions.

Biomacromolecules 2004, 5, 1983.

45. Bulpitt, P.; Aeschlimann, D. New strategy for chemical modification of hyaluronic acid: preparation of functionalized derivatives and their use in the formation of novel biocompatible hydrogels. J. Biomed. Mater. Res. 1999, 47, 152.

46. Elazzouzi-Hafraoui, S.; Nishiyama, Y.; Putaux, J.-L.; Heux, L.; Dubreuil, F.; Rochas, C. The shape and size distribution of crystalline nanoparticles prepared by acid hydrolysis of native cellulose. Biomacromolecules 2008, 9, 57-65. 
47. Mittelbach, P.; Porod, G. Small angle X-ray scattering by dilute colloid systems. The calculation of scattering curves for parallelepipeds. Acta Phys. Austriaca 1961, 14, 185-211. 48. Nayuk, R.; Huber, K. Formfactors of Hollow and Massive Rectangular Parallelepipeds at Variable Degree of Anisometry. Z. Phys. Chem. 2012, 226, 837-854.

49. Pedersen, J. S. Form factors of block copolymer micelles with spherical, ellipsoidal and cylindrical cores. J. Appl. Crystallogr. 2000, 33, 637-640.

50. Pedersen, J. S.; Gerstenberg, M. C. Scattering form factor of block copolymer micelles. Macromolecules 1996, 29, 1363-1365.

51. Beck, S.; Bouchard, J.; Berry, R. Dispersibility in water of dried nanocrystalline cellulose. Biomacromolecules 2012, 13, 1486-1494.

52. Debye, P. Molecular-weight determination by light scattering. The Journal of Physical Chemistry 1947, 51, 18-32.

53. Durand-Gasselin, C. 1.; Capelot, M.; Sanson, N.; Lequeux, N. Tunable and Reversible Aggregation of Poly(ethylene oxide-st-propylene oxide) Grafted Gold Nanoparticles.

Langmuir 2010, 26, 12321-12329. 
TABLE OF CONTENTS GRAPHICS

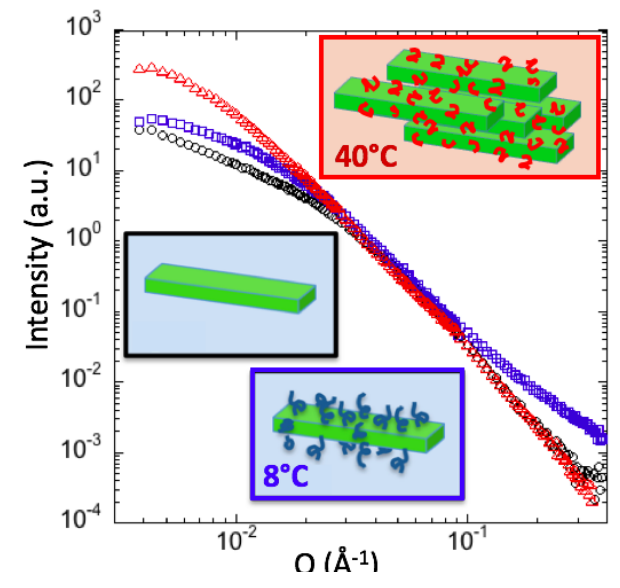

